\title{
Etude sommaire de la répartition des glossines dans l'empire d'Ethiopie (")
}

\author{
par J. BALIS et P. BERGEON
}

\begin{abstract}
RESUME
Les glossines sont présentes dans toutes les régions basses de l'Empire d'Ethiopie quand les conditions de température et d'hygrométrie sont convenables.

Les auteurs ont inventorié les espèces suivantes :

Glossina tachinoides

Glossina fuscipes fuscipes

Glossina morsitans submorsitans

Glossina pallidipes

Glossina longipennis.
\end{abstract}

L'Ethiopie est un vaste pays montagneux situé entre $3^{\circ}$ et $18^{\circ}$ de latitude nord et $33^{\circ}$ et $48^{\circ}$ de longitude. Il est limité à l'ouest par le Soudan, au sud par le Kenya et à l'est par la Mer Rouge et la Somalie.

Du point de vue climatologique les Ethiopiens distinguent 3 types, caractérisés chacun par des genres de vie différents:

La Dega qui comprend les aires géographiques dont l'altitude est supérieure à 2.400 mètres. Ce sont les régions froides du centre du pays où l'on observe parfois des gelées et qui seraient favorables à un élevage intensif.

La Woinadega entourant la précédente et formée par l'ensemble des zones dont l'altitude est comprise entre 2.400 et 1.500 mètres. Le climat y est de type méditerranéen et parfois même subtropical. On y pratique la culture ainsi que l'élevage.

(*) Dans une précédente note (I-2) nous avons publié le résultat provisoire de nos prospections. Depuis lors, de nouvelles observations ont été effectuées tant par nous que par des confrères médecins. Nous avons également pu analyser une bibliographie plus importante. Cette publication est donc un travail plus complet dans lequel on retrouvera comme trame une grande partie du texte de notre note.
Enfin la Kolla occupant environ la moitié de la superficie éthiopienne et correspondant à toutes les parties basses.

Les pluies sont inégalement réparties et conditionnent la végétation; elles sont rares dans l'Ogaden et la plaine des Danakils où l'on observe, particulièrement autour du lac Giulietti, des températures très élevées. Par contre, les précipitations deviennent abondantes et régulières dans l'ouest du pays et font des provinces du Wollega, Illubador et Gemu-Gofades régions verdoyantes où la végétation est souvent luxuriante, spécialement dans les zones basses.

Parallèlement, les insectes $y$ sont beaucoup plus abondants et parmi eux on trouve des glossines. Leur répartition, qui fait l'objet du présent travail, est essentiellement fonction de trois facteurs : altitude, hygrométrie et type de végétation. On en a signalé dans des régions sèches le long de rivières telles que la Webe Shebele ou la Genale, mais il semble que l'ouest du pays soit plus spécialement infesté.

\section{TRAVAUX ANTERIEURS}

Il y a environ 70 ans que la présence de glossines a été signalée en Ethiopie. De nom- 
breux travaux, pour une grande part dûs à des auteurs italiens, en témoignent. DI DOMIZIO, à la suite de ses observations antérieures en Somalie et en Erythrée (15-16-17-18) s'est spécialement intéressé, dès 1929 aux problèmes posés par les glossines et les trypanosomiases. GHIDINI, dans une série d'excellentes publications (23-24-25-26) fit un inventaire entomologique. Son travail fut repris par BETTINI (5) qui s'attacha à préciser les différents moyens à mettre en cuvre pour lutter contre les trypanosomiases animales et leurs vecteurs. Actuellement, six espèces de glossines ont été identifiées :

\section{- Glossina tachinoides}

GHIDINI (23-24-26) l'a observée en 1939 sur le territoire de Gambella et aux confins de l'Ethiopie et du Soudan, le long des rivières Baro et Gilu. OVAZZA (32) en 1956, la retrouve à la frontière soudanaise aux abords de la rivière Akobo.

\section{- Glossina fuscipes fuscipes}

Elle fut récoltée la première fois par BRUMPT (7) en 1901 sur la rivière Omo. GHIDINI en capture quelques exemplaires autour du lac Awassa; l'année suivante, Tarantino (37) la signale le long des affluents de l'Akobo et note la prédominance des femelles parmi les individus capturés. La même année ROETTI (35) étend son aire aux fleuves Didessa et Birbir; enfin, en 1956 OVAZZA la trouve dans la vallée de la Ghibie.

\section{- Glossina morsitans submorsitans}

Elle semble signalée pour la première fois par DI DOMIZIO (19-20-21) en 1937 dans la vallée de la Didessa ainsi que dans les territoires Galla et Sidamo. En 1938, CACCAVELLA (10-11) confirma le fait et, de plus, observa des trypanosomiases bovines dans la région de Lekemti. La mểme année, ROETTI (36) trouve Glossina morsitans submorsitans le long des fleuves Didessa, Birbir, Chibisc, Berberuaha et en bordure de la frontière soudanaise, le long de l'Akobo. Ce cours d'eau est également cité par OVAZZA en 1956.

\section{- Glossina pallidipes}

Elle est accusée de transmettre les trypanosomiases à Trypanosoma vivax, congolense et brucei chez les bovins. En 1913, CHALMERS et KING signalent dans une publication (12) que POWEL et KELLY l'on trouvée au Soudan entre le Nil et le lac Rodolphe sur les rivières Kideppo et Moro-Kinod. PARDACCHI (23) en 1903 la rencontre en Somalie dans la forêt de Bidi. Ceci est confirmé par d'ALBERTIS en 1905, FERRARI en 1907, CROVERI (14) en 1919 et ZAVATTARI en 1937. Au cours de l'année 1936, MOGGRIDGE (30) l'identifia sur la Webe Shebele; d'après lui, elle reste confinée sur un petit espace pendant la saison sèche et s'écarte de 10 kilomètres du fleuve pendant la saison des pluies. ROETTI (35) la trouva en 1938 le long des fleuves Didessa, Birbir et Akobo. Plus récemment enfin, OVAZZA (32) la situe dans la vallée de la Gogeb et le long de la rivière Birbir.

\section{- Glossina austeni}

Aucune mention n'en a été faite sur le territoire éthiopien, mais elle en semble assez proche puisque dès 1903, PARDACCHI (23) la signale en Somalie dans la forêt de Bidi puis MOGGRIDGE (30) en 1936 ainsi que ZAVATTARI (23) en 1937 trouvent Glossina austeni en bordure du fleuve Juba.

\section{- Glossina longipennis}

Elle fut identifiée par CORTI (13) en 1895 parmi le matériel entomologique récolté en 1893 lors des prospections du capitaine BOTTEGO, le long de la rivière Welmal. L'année suivante, GREENFIELD (12) la retrouva en Somalie et en 1895, PEEL (12) la signale en Ogaden dans les régions de Bur Furleh et Biermuddo, le long du fleuve Daghato, affluent gauche du Webe Shebele. En 1904, durant la mission BOURG de BOZAS, c'est BRUMPT (7) qui la voit puis DRAKE-BROKMAN (12) en 1910 , tous deux à proximité de la rivière Juba. D'après ces derniers auteurs, Glossina longipennis attaquerait les animaux surtout la nuit et pour BRUMPT (7) la maladie appelée Aïno en Ogaden est, en réalité, du Nagana transmis par cette espèce.

Enfin en 1938 elle a été signalée par GHIDINI (24) autour du lac Marguerite et aux environs de Soddu.

\section{- Glossina brevipalpis}

Elle est accusée de transmettre Trypanosoma brucei et Trypanosoma congolense. MOGGRIDGE (30) la signale en 1936 le long de la rivière Juba. FRANCHINI (23) la mentionne en 1937 en Somalie italienne. Cette mouche 


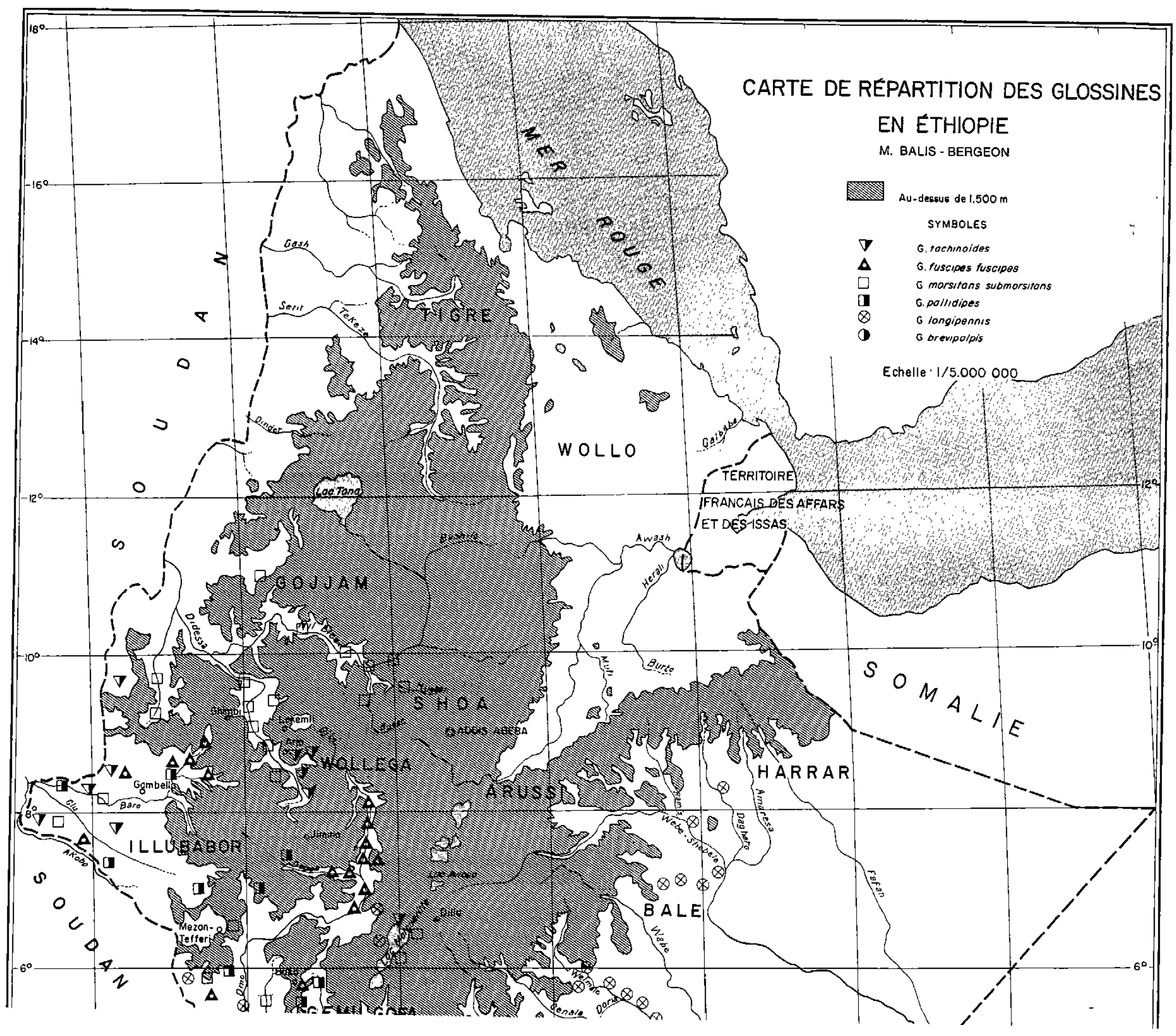


Retour au menu

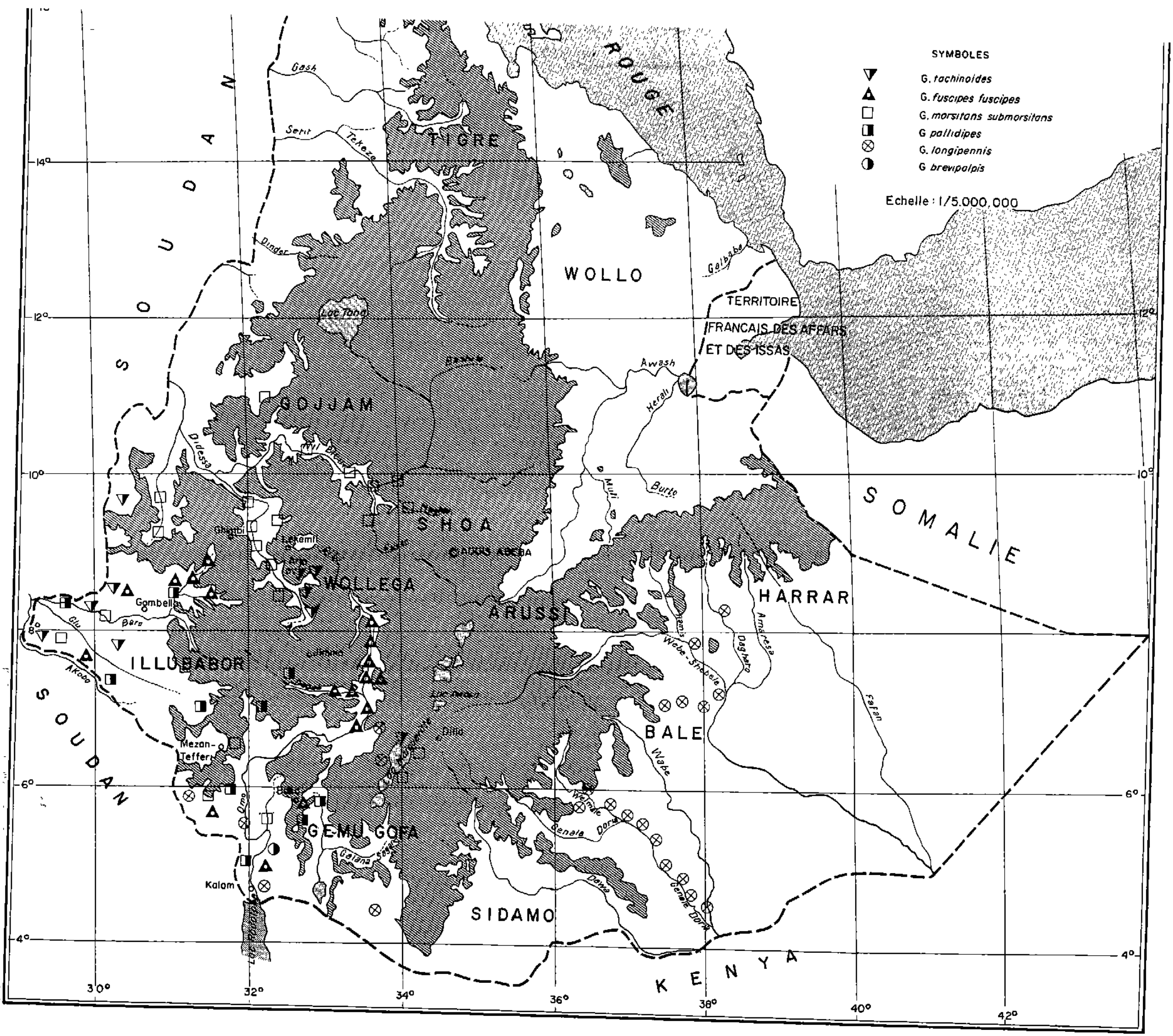


aurait également été trouvée sur les bords de la rivière Omo mais, en définitive, il ne semble pas qu'elle soit présente ou tout au moins abondante sur le territoire éthiopien.

En dehors de ces observations spécialement entomologiques, nous avons consulté un certain nombre de rapports de tournées émanant surtout d'experts de la F.A.O.; c'est ainsi que BANNISTER (3) en 1957, parlait de pertes considérables dues à la trypanosomiase, sur le bétail en provenance du Wollo. C'est également l'avis de PECK (33) qui constatait en 1959 que la zone à glossines s'étendait dangereusement et que de vastes territoires tels que le Wollo devenaient inhospitaliers pour le bétail. Dans le même rapport il affirmait qu'une grande partie de l'ouest éthiopien était interdite à l'élevage à cause des tsé-tsé et de la trypanosomiase qu'elles transmettent. Ce même auteur, dans un autre rapport (35) écrivait avoir vu des glossines et des animaux malades dans la province de Gojjam et selon lui, la zone infestée s'étendait jusqu'à LEKEMTI.

En 1961 OTTE (31) constatait que la trypanosomiase prenait une importance de plus en plus grande en Ethiopie et envisageait l'éventualité d'un contrôle des régions à glossines. Pour MacPHERSON (29) de vastes zones basses sont abandonnées par les éleveurs qui migrent tous vers les régions plus élevées, à l'abri des glossines. Enfin, un rapport du LIVESTOCK DIVISION (28) datant de 1962 signalait que la trypanosomiase était un sérieux problème dans la vallée de l'Omo et spécialement à l'ouest du district de Kambete. Le Gemu-Gofa était atteint, surtout le long de la rivière Sagan. Les provinces du Bale et de l'Arussi ainsi que le Chercher étaient également infestés.

L'ensemble de ces observations nous montre que le problème des glossines et de la trypanosomiase n'est pas une nouveauté en Ethiopie mais ces études sont disparates. Nous avons cependant retrouvé une carte établie par l'entomologiste POTTS, qui réalise une synthèse intéressante, particulièrement dans l'Ogaden et la Somalie. Enfin, il semble que toutes les régions dont l'altitude est inférieure ou égale à 1.500 mètres hébergent des glossines, pourvu que le type de végétation et le degré hygrométrique s'y prêtent. L'Erythrée ne doit pas remplir ces conditions car BEZZI (6) et FERRARO (22) au cours d'enquêtes approfondies sur les diptères hématophages, n'ont jamais pu découvrir de glossines. Cette observation doit également être valable en ce qui concerne la région des Dancalis car SEGUY (36) n'en parle pas dans son étude sur les diptères, effectuée au cours de l'expédition du Baron FRANCHETTI.

\section{TRAVAUX PERSONNELS}

Nous avons identifié les glossines, d'une part avec la publication de BARROS MACHADO (4) pour les mouches du groupe palpalis, d'autre part avec les ouvrages de ZUMPT (38) et BUXTON (9) pour celles du groupe morsitans.

Nos récoltes ont d'abord été faites au cours de tournées en compagnie de confrères ou d'agents du Service de l'élevage occupant différents postes de brousse. Leur aide nous a été précieuse grâce à leur grande connaissance du pays et aux observations qu'ils avaient déjà réalisées au cours de leurs multiples déplacements. C'est ainsi que nous avons exploré avec le Docteur KOVALENKO en décembre 1965, une partie des rives des fleuves Didessa et Angar dans la région du Lekemti. Début janvier 1966, c'est en compagnie des Docteurs BLANC et POUSTIS que furent prospectés les environs du lac Marguerite et nous sommes remontés jusqu’à Hagare Mariam. Fin janvier et début février, avec l'assistant d'élevage SHOA SAGUET, des recherches ont été faites sur une partie des cours des rivières Gibi et Gogeb. Enfin l'un de nous, en avril 1967 et janvier 1968, a ramené des glossines des provinces du Gemu-Gofa et du Wollega.

En plus de ces récoltes personnelles, nous avons reçu des échantillons collectés par les personnes suivantes que nous remercions :

- Docteurs BLANC et GRATTEAU. Province du Gemu-Gofa, le long de l'Omo en bordure du Kenya. Rivière Maze, affluent de l'Omo Bottego. Rivières Oito, Gorgora et Bako ainsi qu'aux environs de Kayafer et Bako.

- Docteur KOVALENKO. Province du Wollega, vallée du Nil Bleu dans le district d'Amourou à environ 90 kilomètres de Debre Marcos. Rivière Birbir, route de Lekemti à Ghimbi, fleuve Dabous, rivière Jokyl (affluent de la Darana), rivière Shebel à la limite de l'Ethiopie et de la plaine soudanaise, rivière Keto (affluent du Birbir) dans le district de Dembidollo. 
- Docteur POUSTIS. Village de Wanago, près du lac Marguerite et le long du torrent coupant la route venant de Dilla.

- Docteur HOUIN. Vallée de l'Omo à une trentaine de kilomètres au nord de Kalam.

- Docteurs HAUBNEY et GIRMA. Rivière Gila dans l'Illubabor.

- Service de l'élevage de Mesan Tefferi dans la province de Kaffa.

- AYEle BerU. Province du Gemu Gofa, rivière Cullufu (affluent du lac Marguerite) dans le district d'Arbaninch.

- Asfao TEgene. Province de Shoa, village de Sollagerbi sur la rivière Annonu, affluent de la Guder, village de Dero, le long de la rivière Muger.

- SHOA SAGUET. Province de Kaffa, le long de la Shata (petite rivière se jetant dans la Cogeb).

- Docteur COLSON, route de Lekemti à Angar.

- Docteur TAGER KAGAN. Lac Shamo et rivière Sagan.

Toutes ces glossines ont été examinées au laboratoire et appartiennent à quatre espèces:

- Glossina tachinoides;

- Glossina fuscipes fuscipes;

- Glossina morsitans submorsitans;

- Glossina pallidipes.

\section{- Glossina tachinoides}

Nous l'avons rencontrée sur les bords de la Didessa au village de Loko, sur le prolongement de la route allant de Lekemti à Arjo. A cet endroit, le fleuve est bordé par une galerie forestière dense hébergeant les mouches. Elles ne s'en éloignent pas au-delà d'une centaine de mètres et piquent à partir de 4 heures de l'après-midi. Nous en avons capturé 7 ( 5 mâles et 2 femelles). Elles sont également présentes aux environs du village de Wanago à 16 kilomètres à l'ouest de Dilla près du lac Marguerite (un mâle capturé en octobre 1966), ainsi que le long de la rivière Takaw dans l'Illubabor (12 mâles et 20 femelles en mai 1966).

\section{- Glossina fuscipes fuscipes}

Elle est relativement abondante en Ethiopie et fut trouvée aux endroits suivants :

- Dans la province du Gemu-Gofa, le long des rivières Maze ( 2 mâles, 2 femelles), Gorgora (1 femelle), Bazo (1 mâle, 1 femelle), aux envi- rons de Kalam ( 7 mâles, 8 femelles), enfin entre Cuccia et Sodou ( 2 mâles) à l'altitude de 1.500 mètres.

- D'après le Docteur BLANC, les glossines sont extrêmement abondantes aux abords de tous les cours d'eau de la région y compris l'Omo Bottego. Le long de la rivière Keto (affluent du Bir Bir), dans le district de DembiDollo et la province de Wollega (4 mâles, 2 femelles).

- Le long de la rivière Shebel (altitude 1.450 mètres) à proximité du village de Shebel dans le district d'Artillo et la province du Wollega (3 mâles, 1 femelle).

- Le long du Bir Bir aux environs du village de Degono à l'altitude de 1.420 mètres (2 mâles).

- Sur la rivière Shata (petite rivière se jetant dans la Gogeb à proximité du village de Tercia (4 mâles, 2 femelles).

- Sur la rivière Gibi à l'endroit où elle croise la route d'Addis Abeba à Jimma; la vallée assez encaissée est à l'altitude de 1.340 mètres.

\section{- Glossina morsitans submorsitans}

C"est une espèce très répandue en Ethiopie; nous l'avons rencontrée :

- Dans la sous-province de Metekel (19 mâles, 1 femelle).

- Sur les bords de la Didessa dans la galerie forestière ainsi que dans la savane qui lui fait suite jusqu'à 10 kilomètres du fleuve ( 9 mâles, 5 femelles), l'altitude moyenne est de 1.300 mètres.

- A proximité de la rivière Angar à une altitude d'environ 1.300 mètres $(5$ mâles, 3 femelles). Le bétail n'existe pratiquement pas dans ces régions basses, les éleveurs ayant émigré vers les hauteurs.

- Sur la route de Lekemti à Angar (13 femelles, 11 mâles) à l'altitude de 1.600 mètres.

- Autour du lac Marguerite aux environs des villages de Wanago et Ledo ainsi que sur la rivière Cullufu à une altitude de 1.160 mètres (4 mâles, 4 femelles). Les glossines n'y étaient pas très abondantes et on y trouvait du bétail plus ou moins trypanosomé. Cette région est souvent marécageuse et les habitants sont presque tous fortement impaludés.

- A proximité du Nil Bleu, au village d'Amourou dans la sous-province de Shambo (3 mâles, 2 femelles). 
- Aux environs du village de Dero, sur la Mugher. Cette dernière coule au fond d'une vallée très encaissée dont l'altitude est inférieure à 1.500 mètres ( 1 mâle, 4 femelles).

- Le long de la rivière Annonu dont la vallée est du même type que la précédente. Les éleveurs ont émigré sur les hauteurs et ne descendent que très rarement ( 8 mâles, 4 femelles).

- Sur la route de Lekemti à Ghimbi à 1.250 mètres au pont de la Didessa à 11 heures du matin, entre les rivières Didessa et Jokyl ainsi que le long de cette dernière, à l'altitude de 1.350 mètres ( 25 mâles, 3 femelles).

- Le long du fleuve Dabous (10 mâles, 8 femelles).

- Le long de la rivière Gilu dans la province de l'Illubabor (2 mâles).

- Le long de la rivière Baro près de Gambella dans l'Illubabor à une altitude de 800 mètres ( 9 mâles).

- Dans la savane de Tourmi près de la rivière Milti à une altitude de 900 mètres (1 mâle).

- A Mesan-Tefferi dans la province de Kaffa à une altitude de 1.400 mètres (14 mâles, 15 femelles).

\section{- Glossina pallidipes}

Les exemplaires que nous possédons proviennent uniquement de la province du Gemu Gofa.

Les Docteurs HOUIN et BAZIN ont capturé 4 mâles et 3 femelles à une trentaine de kilomètres au nord de Kalam, dans la vallée de l'Omo, à proximité d'une forêt galerie peu épaisse. D'après ces auteurs (25) certains endroits étaient infestés à un point tel qu'on ne pouvait y séjourner dans la journée.

C'est également l'opinion des Docteurs BLANC et GRATTEAU qui ont trouvé Glossina pallidipes en abondance sur la rivière Otto (14 mâles, 8 femelles) aux environs de Kayafer ( 1 femelle) à l'altitude de 1.550 mètres et entre cette dernière localité et Bako (1 mâle, 1 femelle).

Le Docteur TAGER KAGAN l'a rencontrée aux abords du lac Shamo et de la rivière Sagan (3 femelles, 5 mâles).

\section{Interprétation des résultats}

Cet inventaire est forcément incomplet car il est certain que d'autres gîtes à glossines existent dans des régions dont l'altitude est inférieure à 1.500 mètres et où l'on trouve la végétation et l'hygrométrie favorables. C'est ainsi que les vallées de l'Awash et du fleuve Tekasse sont pour le moins suspectes puisque des frottis en provenance de cette région étaient positifs à Trypanosoma vivax. Il y aurait des glossines à une trentaine de kilomètres de Bardar, c'est-àdire assez près du lac Tana. Ceci n'aurait rien d'étonnant puisqu'en 1966 on a relevé 14 foyers de trypanosomiase dans la province du Gojjam.

En tenant compte de tous ces éléments nous avons dessiné une carte de répartition sur laquelle tous les territoires dont l'altitude est supérieure à 1.500 mètres sont figurés en sombre. Les contours ont été obtenus par assemblage des réductions photographiques de 39 cartes au 1/500.000 sur lesquelles les courbes de niveau étaient figurées.

A l'examen de cette carte, on se rend compte qu'une grande partie du territoire éthiopien est infestée et les hautes terres (Dega et Woinadega) qui devraient être à l'abri des trypanosomiases sont pénétrées profondément par des vallées encaissées servant de refuge aux glossines. En outre, l'ouverture récente de routes carrossables a pour résultat le transport par véhicules automobiles des insectes. C'est ainsi que des glossines sont amenées jusqu'à Lekemti où elles peuvent contaminer du bétail vivant à 2.000 mètres et, de là, regagner les basses terres : ce fait expliquerait l'envahissement progressif de la région de Sire. Le Docteur COLSON a d'ailleurs recueilli Glossina submorsitans à l'altitude de 1.600 mètres sur la route de Lekemti à Angar. Les zones de sécurité sont donc considérablement réduites.

Ces remarques sont importantes car il serait possible de récupérer de vastes étendues de pâturages en mettant en culture les vallées contaminées. L'opération serait doublement rentable. Nous pensons que, dans ce cas précis et uniquement par des mesures agronomiques, ne nécessitant aucune aide extérieure, un projet d'éradication définitive de la trypanosomiase dans les hautes terres aurait de réelles chances d'aboutir.

Pour terminer, signalons que nous n'avions jamais observé ni entendu parler de trypano- 
somiase humaine jusqu'en 1967, époque à laquelle 3 cas dus à Trypanosoma rhodesiense ont été signalés dans l'ouest du pays: l'un à Bako par un médecin américain du Corps de la Paix, le Docteur MAYIE, les deux autres à Gambella, par l'équipe Namru III (Naval Medical Research Unit $n^{10}$ 3) sous la direction du Docteur D. C. KENT.

Enfin, il y a 6 mois le Docteur CHALES, Directeur de 1'O.M.S. en Ethiopie, estimait le nombre de cas humains à 67 ; c'est dire ]'extension que semble prendre la maladie.

Dans ces régions, les Ethiopiens fuient devant l'envahissement progressif des glossines et sont remplacés petit à petit par des populations noires venues du Soudan, dont certains éléments sont vraisemblablement atteints de maladie du sommeil. C'est là un gros danger car les vecteurs actifs existent en abondance et il serait souhaitable que du point de vue humain, des mesures prophylactiques soient envisagées sérieusement.

\section{CONCLUSION}

Les résultats obtenus par notre enquête et l'utilisation des travaux antérieurs nous ont permis d'établir une carte provisoire de répartition des glossines dans l'Empire d'Ethiopie. On peut considérer qu'il existe les espèces suivantes :

- Glossina tachinoides,

- Glossina fuscipes fuscipes,

- Glossina morsitans submorsitans,

- Glossina pallidipes,

- Glossina longipennis.

Glossina brevipalpis ne semble pas présente en Ethiopie.

Il serait important de poursuivre ce travail. Plusieurs vallées telles que celles du. Nil Bleu, de la Didessa et de l'Omo pourraient faire l'objet d'un inventaire détaillé en vue d'un projet d'éradication des trypanosomiases animales dans les régions de moyenne altitude.

\section{SUMMARY}

Succint study of the Glossina distribution in Ethiopian Empire

Glossina are found in all the lower regions of Ethiopian Empire when the conditions of temperature and hygrometry are appropriate.

The authors have inventoried the following species:

Glossina tachinoides

Glossina fuscipes fuscipes

Glossina morsitans submorsitans

Glossina pallidipes

Glossina longipennis

\section{RESUMEN}

Estudio sumario de la repartición de las glosinas en el Imperio de Etiopia

Se encuentran las glosinas en todas las regiones bajas del Imperio de Etiopa cuando las condiciones de temperatura y de higrometria son buenas.

Los autores inventariaron las especies siguientes:

Glossina zachinoides

Glossina fuscipes fuscipes

Glossina morsitans submorsitans

Glossina pallidipes

Glossina longipennis.

\section{BIBLIOGRAPHIE}

1. BALIS (J.), BERGEON (P. H.), «Tsetse fly study in Ethiopia ", Preliminary note. International Scientific Council for Trypanosomiasis Research, Eleventh meeting Nairobi 1966. Publication $n^{\circ} 100$, p. 115.

2. BALIS (J.) et BERGEON (P.), «Etude de la répartition des glossines en Ethiopie », Bull. Org. Mond. Santé, 1968, 38: 809-813.
3. BANNISTER (C.L.), «Report Nr. 688 to the Government of Ethiopia on Livestock disease control \%, Rome, 1957 , p. 4.

4. BARROS MACHADO (A.), "Révision systématique des glossines du groupe palpalis », Publication du Museu do Dundo. Angola, 1954.

5. BETTINI (T. M.), «L'importanza della lotta contro le glossine (glossina spp) quala profilassi di 
aloune tripanosi dell' AOI », $A$ grti, col., 1941. p. $374-415$ et $416-423$.

6. BEZZl (M.), "Materiali per la conoscenza della fauna eritrea raccolti da Magretti. Ditteri », Boll. Soc. Entomol. Ital. 1901, 33.

7. BRUMPT (E.), «Statistique médicale faite dans un voyage à travers l'Afrique tropicale (note préliminaire) ", C.R. de l'Ass. franc. pour l'Avanc. des Sciences; Congrès d'Angers 1903.

8. BRUMPT (E.), « La maladie désignée sous le nom d'Aïno par les Somalis de l'Ogaden est une trypanosomiase probablement identique au Nagana de l'Afrique Orientale », C.R. séanc. Soc. Biol. 1904, 61: 673 .

9. BUXTON (P.A.A.), « The natural history of tsetse flies », London, Tropical School of Medicine, 1955 .

10. CACCAVELla (A.), « Prime osservazioni sulle infezioni degli anmali domestici dell' Uollega. Profillassi e entomologia», Nuova Veterinaria 1938 (4) : 13-17.

11. CACCAVElLA (A.). « Nota preliminare sulle trypanosomiasi del occidente ethiopico", Riv. Biol. Col., 1939, 2.

12. CHALMERS (A. I.), KING (H. H.), « Distribution of Glossina longipennis Corti $», J$. Trop. Med. Hyg. 1913, $16: 320-322$.

13. CORTI (E.), «Esplorazione del Giuba e dei suci affluenti compiuta dal cap. V. Bottego », Ann. Mus. Clv. Genova 1895, 15 : 129-148.

14. CROVERI (P.), « Osservasioni sulla biologia della Glossina pallidipes della Somalia Italiana e sutla transmissione agli animali domestici della tripanosi detta "Ghendi" ", Ann. Igien. 1919, 29 : 432-447.

15. DI DOMIZIO (G.). «I tripanosomi patogeni del bestiame nella Somalia ltaliana con particolare riguardo al Trypanosoma congolense », Rimnov. Med., 1929, 7.

16. DI DOMIZIO (G.), "Sul tripanosoma cazalboui in colonia Fritrea e sulla relativa tripanosi », Miss. Scient. per l'Eritrea, 1930.

17. DI DOMIZIO (G.), «A proposito delle glossine nella Somalia Italiana », III Congr. Naz. Med. Col., Tripoli, 1937.

18. DI DOMIZIO (G.), «A proposito delle glossine nella Somalia Italiana", Boll. Soc. Ital. Med. Igten. Col., 1931.

19. DI DOMIZIO (G.), «Nagana nella Valle de] Didessa in Abissinia ", Arch. Ital. Sci. Med. Col. Parass., 1937, $18: 129-130$.

20. DI DOMIZIO (G.), «Nagana nella Valle del Didessa in Abissinia (seconda nota) », Arch. Ital. Sci. Med. Col. Parass. 1937, 18 : 193-195.
21. DI DOMIZIO (G.), «Sul Nagana degli equini nella Valle del Didessa in Abissinia ", Arch. Jal. Sci. Med. Col. Parass. 1937, 18.

22. FERRARO (G.), «Ditteri ematofaghi della colonia Eritrea incriminati della transmissione delle tripanosomiasi locali ». Clinica veterinaria Rassegna di Polizia Sanitaria et di Igiene, Septembre 1917.

23. GHIDINI (G. M.), «Le glossine dell'Africa orientale Italiana », Riv. Bıol. colon. 1938, vol. 1, 16: 53-79.

24. GHIDINI (G. M.), « Nuovi dati sulla distribuzione delle glossine nelle terre dell' Impero $\%$, Riv. Biol. colon. 1939, vol. 2, 329.

25. GHIDINI (G. M.), "Di alcuni Ditteri ematofagi della regione dei Laghi (AOI) », Boll. Soc. Entomol. Ital. Genova, 1939, 71 : 40 .

26. GHIDIN] (G. M.), «Glossine e tabanidi dell' AOI \%. Annali Museo civico storia naturale. 1939, 58 : 339.

27. HOUIN (R), BAZIN (J. C.), «Expédition française de l'Omo. 1967 », Service médical. Rapport d'activités.

28. LIVESTOCK DIVISION, * Report on livestock (cattle) survey in southern Ethiopia 1961-62\%, Addis Abeba, déc. 1962.

29. MACPHERSON, "Report no 1813 on the control of animal diseases ", Rome, 1964, p. 8.

30. MOGGRIDGE (J. Y.), "Some observations on the seasonal spread of Glossina pallidipes in Italian Somaliland with notes on $G$. brevipalpis and G. austeni ", Bull. ent. Res., 1936. $27: 449-466$.

31. OTTE (E.), "Report nr. 1426 on the control of Livestock diseases *, Rome, 1961, p. 13.

32. OVAZZA (M.), «Contribution à l'étude des diptères vulnérants de l'Empire d'Ethiopie, IV Glossines ", Bull. Soc. Path. Exot. 1956 (1) : 204209.

33. PECK (E. F.), * Report nr. 1447 on the Animal Diseases Control », 1959, p. 6.

34. PECK (E.F.), «An adcount of a journey to Gojjam province from 29th May to Ist July $1959 »$.

35. ROETTI (C.), « La tripanosomiasi animale neI Galla e Sidama », Arch. ital. Sci. nied. colon. parasit. 1938, $19: 335$.

36. SEGUY (E.), «Insectes diptères. Spedizione del Barone Franchetti in Dancalıa », Annali Mus. Civ. Stor. Nat. Giacomo Doria, 1930-31, 55.

37. TARENTINO (G B), «La tripanosomiasi nel Galla e Sidama », Riv. Biol. col . 1938, $1: 161-164$.

38. ZUMPT (F.), « Die Tsetsefliegen ». Jena, Fischer, 1936. 